\title{
Original
}

\section{Susceptibility of the Wnt/p-catenin Pathway Accelerates Osteogenic Differentiation of Human Periodontal Ligament Stem Cell Spheroids}

\author{
Takuya Toshimitsu ${ }^{1,2)}$, Hiroshi Kajiya ${ }^{2,3)}$, Madoka Yasunaga ${ }^{2,4)}$, Munehisa Maeshibaa $^{2,5)}$, Seiichi Fujisaki ${ }^{2,6)}$, Naoyuki Miyaguchi ${ }^{2,6)}$, \\ Masahiro Yamaguchi ${ }^{2,7)}$, Hidefumi Maeda ${ }^{8)}$, Hiroshi Kojima ${ }^{1)}$ and Jun Ohno ${ }^{2)}$ \\ 1) Dentistry for the Disabled, Department of Oral Growth and Development, Fukuoka Dental College, Fukuoka, Japan \\ 2) Research Center for Regenerative Medicine, Fukuoka Dental College, Fukuoka, Japan \\ 3) Section of Cellular Physiology, Department of Physiological Science and Molecular Biology, Fukuoka Dental College, Fukuoka, Japan \\ 4) Section of Orthodontics, Department of Oral Growth and Development, Fukuoka Dental College, Fukuoka, Japan \\ 5) Division of Removable Prosthodontics, Department of Oral Rehabilitation, Fukuoka Dental College, Fukuoka, Japan \\ 6) Division of Oral Implantology, Department of Oral Rehabilitation, Fukuoka Dental College, Fukuoka, Japan \\ ${ }^{7)}$ Section of Geriatric Dentistry, Department of General Dentistry, Fukuoka Dental College, Fukuoka, Japan \\ ${ }^{8)}$ Division of Oral Rehabilitation, Department of Endodontology and Operative Dentistry, Faculty of Dental Science, Kyushu University, Fukuoka, \\ Japan
}

(Accepted for publication, January 15, 2019)

\begin{abstract}
Human periodontal ligament stem cells (HPLSCs) contribute to the regeneration of periodontal tissue because of their multilineage potential. Although monolayer cultures are commonly used in cell cultures, they inadequately overcome their low differentiation capacity. The use of spheroid cultures is expected to overcome the problem of mimicking the in vivo microenvironment. In this study, we assessed whether HPLSC spheroids are susceptible to osteogenic differentiation through the canonical Wnt/ $\beta$-catenin signaling pathway. HPLSC spheroids were generated using low-binding plates. Osteogenic differentiation of monolayer- and spheroid-derived HPLSCs was induced by osteogenic induction medium. Increased expression levels of osterix and Runx2 and intense staining of alkaline phosphatase (ALP) activity were observed in spheroid-derived HPLSCs, as compared with monolayer-derived cells. During spheroid formation, the integrin pathway of cells composed of spheroids was activated through focal adhesion kinase (FAK), suggesting that this activation may induce susceptibility of the Wnt pathway in HPLSC spheroids. Wnt 3a stimulation increased the expression levels of $\beta$-catenin and T-cell factor (TCF), but decreased that of glycogen synthase kinase-3 $\beta$. Wnt 3a-induced the expression of $\beta$-catenin and TCF was effectively decreased by Dickkopf-1 (Dkk-1), a Wnt antagonist. Wnt 3a stimulation also increased the expression levels of osterix and Runx2 which was accompanied by intense ALP staining, in HPLSC spheroids, whereas the addition of Dkk-1 decreased both expression levels and ALP staining. These findings indicate that HPLSC spheroids enhance osteogenic differentiation because cells, composed of spheroids, induce susceptibility of the canonical Wnt pathway, which is mediated by activation of the integrin/FAK pathway during spheroid formation.
\end{abstract}

Key words: Osteogenesis, Human periodontal ligament stem cells, Wnt3a, $\beta$-catenin, Spheroids, Integrin

\section{Introduction}

Human adult mesenchymal stem cells (MSCs) are a population of multipotent progenitor cells present in bone marrow and most connective tissues with the potential to differentiate into various cell types, such as osteoblasts, chondrocytes, and adipocytes ${ }^{1,2)}$. Tooth-related tissues are extremely rich sources of multipotent MSCs, especially human periodontal ligament stem cells (HPLSCs) have the potential to differentiate into osteoblasts and adipocytes ${ }^{3)}$. Hence, HPLSCs have been investigated for therapeutic regeneration of bone and periodontal tissues ${ }^{2-4)}$. Therefore, it is important to understand the molecular mechanisms underlying the osteogenic differentiation of HPLSCs.

Although MSCs are generally cultured as two-dimensional (2D) monolayers, the three-dimensional cell culture technique has been used

Correspondence to: Dr. Jun Ohno, Research Center for Regenerative Medicine, Fukuoka Dental College, 2-15-1 Tamura, Sawara-ku, Fukuoka, Fukuoka 8140193, Japan; Tel: +81928010411 (Ext 684); Fax: +81928014909; E-mail:johno@college.fdcnet.ac.jp widely in many fields of biomedical research. In comparison with conventional 2D monolayer cultures, which are unable to construct the normal cell-cell interactions present in vivo, cells in spheroids more closely mimic the in vivo situation with regard to differentiation patterns and spatial cell-cell and cell-extracellular matrix (ECM) interactions ${ }^{5,6}$. These advantages have led to the increasing use of spheroids as models for studies of differentiation and tissue morphology $y^{7,8)}$.

The initial step of the formation of multicellular spheroids is involved in cell-cell contact due to cell aggregation. Several reports have described the roles of cadherins and integrins in the process of spheroid formation $^{9-11)}$. Many cell-cell and cell-EMC interactions are regulated by integrins, a large family of proteins that function as heterodimeric transmembrane receptors of $\alpha$ - and $\beta$-subunits and act to link cells to ECM proteins ${ }^{12,13)}$. The integrin $\alpha 5 \beta 1$ mediates strong cohesively in cellular aggregates of spheroids ${ }^{9)}$. Because integrins allow osteoblasts to interact with the ECM and mediate the differentiation of MCSs into osteoblasts $^{14,15)}$, activation of the integrin signaling pathway of MSC sphe- 
roids is thought to accelerate osteogenic differentiation.

Activation of the integrin pathway facilitates activation of the canonical Wnt/ $\beta$-catenin signaling pathway ${ }^{16}$. Wnt signaling pathway is responsible for a variety of cellular processes including fate specification, establishment of polarity, proliferation, migration, and tissue morphogenesis ${ }^{17}$. A growing body of evidence suggests that the canonical $\mathrm{Wnt} / \beta$-catenin pathway may play an important role in regulating osteogenic differentiation of MSCs ${ }^{18-24}$. As an effect of the activated canonical Wnt pathway on enhancement of osteogenesis, binding of canonical Wnt proteins to Frizzled receptors and the LRP5 and LRP6 co-receptors leads to the inhibition of glycogen synthase kinase $3 \beta$ (GSK-3 $\beta$ ) and decreased degradation of $\beta$-catenin. Stabilization of $\beta$-catenin leads to its translocation into the nucleus where, once associated with the TCF/LEF transcription factors, it triggers the transcription of osteogenic gene. Therefore, we hypothesized that activation of the integrin pathway in MSC spheroids will lead to the enhancement of osteogenic differentiation mediated by the canonical $\mathrm{Wnt} / \beta$-catenin pathway.

Thus, the purpose of this study was to test the hypothesis that susceptibility of the canonical Wnt/ $\beta$-catenin signaling pathway can accelerate osteogenic differentiation of HPLSC spheroids. To test this hypothesis, several aims were developed to determine whether HPLSC spheroids represent activation of the integrin pathway and enhancement of osteogenic differentiation through integrin activation of the canonical $\mathrm{Wnt} / \beta$-catenin signaling pathway.

\section{Materials and Methods \\ Reagents and antibodies (Abs)}

Fetal bovine serum (FBS) was purchased from HyClone Laboratories Inc. (South Logan, UT, USA). Dulbecco's modified Eagle's medium (DMEM) was purchased from Fujifilm WakoPure Chemical Co. (Osaka, Japan), while $1 \times$ antibiotic-antimycotic solution ( $1 \times$ Anti-Anti), $0.25 \%$ trypsin/ethylenediaminetetraacetic acid (EDTA), and goat anti-rabbit immunoglobulin G-Alxa Flour 488 were obtained from Invitrogen Corporation (Carlsbad, CA, USA). Hoechst 33324 nucleic acid stain, an alkaline phosphatase (ALP) kit, and monoclonal $\mathrm{Ab}$ against $\beta$-actin were purchased from Sigma-Aldrich Corporation (St. Louis, MO, USA). The Pierce ${ }^{\mathrm{TM}}$ BCA Protein Assay Kit was obtained from Thermo Fisher Scientific (Rockford, IL, USA). Rabbit anti-Sp7/osterix, and rabbit anti-runt-related transcription factor 2 (Runx2) were obtained from Abcam Inc. (Cambridge, UK). Rabbit anti-focal adhesion kinase (FAK), rabbit monoclonal anti-phospho-FAK (Tyr397, p-FAK), rabbit anti-integrin $\beta 1$, rabbit anti-glycogen synthase kinase $3 \beta($ GSK-3 $\beta$ ), rabbit anti-T-cell factor (TCF), and rabbit anti- $\beta$-catenin were purchased from Cell Signaling Technology (Danvers, MA, USA). Recombinant human Wnt 3a protein was obtained from R\&D Systems, Inc. (Minneapolis, MN, USA). Recombinant human Dickkopf-1 (Dkk-1) was purchased from Funakoshi Co., Ltd. (Tokyo, Japan).

\section{Cell culture}

HPLSCs were stored at the Department of Endodontology and Operative Dentistry, Division of Oral Rehabilitation, Faculty of Dental Science, Kyushu University ${ }^{2}$. Cryopreserved HPLSCs were thawed and plated onto a tissue culture dish maintained in DMEM supplemented with $10 \%(\mathrm{v} / \mathrm{v})$ FBS and $1 \times$ Anti-Anti solution, then dissociated with $0.25 \%$ trypsin/EDTA, counted, and either re-plated for monolayer culture or used for spheroid formation.

\section{Spheroid formation of HPLSCs}

Dissociated HPLSC monolayers were responded in medium to ob- tain a single cell suspension. HPLSCs $\left(1 \times 10^{5}\right.$ cells $/ \mathrm{ml}$, corresponding to approximately 10,000 cells/well) were added to the wells of Corning ${ }^{\circledR} 96$ well Ultralow Attachment microplates (Corning Inc., Corning, NY, USA) and incubated in medium at $37^{\circ} \mathrm{C}$ for $24 \mathrm{~h}$.

\section{Osteogenic differential of monolayer- and spheroid-derived HPLSCs, and HPLSC spheroids}

To generate spheroid-derived HPLSCs, HPLSC spheroids, after $24 \mathrm{~h}$ of suspension culture in Ultralow Attachment microplates, were washed three times with phosphate-buffered saline (PBS) and incubated with $0.25 \%$ trypsin-EDTA solution at $37^{\circ} \mathrm{C}$ for $15 \mathrm{~min}$ with mechanical agitation until a single cell suspension was obtained. Dissociated cells were plated and cultured until reaching confluence. Confluent monolayers, spheroid-derived HPLSCs and HPLSC spheroids were cultured in osteogenic induction medium (OIM), which was composed of DMEM supplemented with $100 \mathrm{nM}$ dexamethasone, $50 \mu \mathrm{M}$ ascorbic acid, $10 \mathrm{mM}$ $\beta$-glycerophosphate, and 10\% FBS. The OIM was replaced three times each week. For a Wnt 3a-stimulation experiment, confluent cells and HPLSC spheroids were incubated with OIM alone, a mixture of OIM and Wnt 3a, or a mixture of OIM, Wnt 3a (50 ng/ml), and Dkk-1 (250 $\mathrm{ng} / \mathrm{ml}$ ) for 1 week.

\section{ALP staining}

HPLSC spheroids stimulated by Wnt3a with or without Dkk-1 were frozen using OCT compound. Frozen sections of HPLSC spheroids were used for ALP staining. Monolayer and spheroid-derived HPLSCs as well as frozen sections of spheroids were stained with an ALP kit containing Fast Red Violet solution and naphthol AS-BI phosphate solution, in accordance with the manufacturer's instructions. Briefly, cells and sections were fixed with citrate-acetone-formaldehyde for $30 \mathrm{~s}$, rinsed with distilled water (DW), then incubated with alkaline-dye mixture for $15 \mathrm{~min}$ and washed again with DW. The reaction product was evaluated by microscope.

\section{Western blot analysis}

Monolayer- and spheroid-derived HPLSCs as well as HPLSC spheroids were lysed in cell lysis buffer containing $1 \times$ protease/phosphatase inhibitor cocktail. Protein concentrations were measured with the Pierce BCA Protein Assay Kit. Equal amounts $(15 \mu \mathrm{g})$ of protein along with a protein marker (Precision Plus Protein ${ }^{\mathrm{TM}}$ Western $\mathrm{C}^{\mathrm{TM}}$ Standards; BioRad Laboratories, Hercules, CA, USA) were separated on Mini-PROTEAN $^{\circledR}$ TGX $^{\mathrm{TM}}$ Precast Gels for $30 \mathrm{~min}$ at $200 \mathrm{~V}$. The Trans-Blot ${ }^{\circledR}$ Turbo Transfer System (Bio-Rad Laboratories) was used to transfer the separated proteins to a polyvinylidene fluoride membrane. Western blots were processed using the iBind ${ }^{\mathrm{TM}}$ Western System (Life Technologies, Carlsbad, CA, USA) with primary Abs and horseradish peroxidase-conjugated secondary Abs. $\beta$-actin was used as a loading control. An enhanced chemiluminescence system (SignalFire Plus ECL Reagent; Cell Signaling Technology(Danvers, MA, USA)) was used to develop the protein bands. The protein levels were quantified by densitometry using the ImageQuant ${ }^{\mathrm{TM}}$ LAS 4000 biomolecular imager (GE Healthcare, Uppsala, Sweden). Band densities are presented as fold-increases of the expression levels of primary Abs (normalized to $\beta$-actin) and compared with the results of the control. Quantification results are shown below the corresponding blots. The densitometry data were from a representative of three independent experiments.

\section{Immunocytochemical assay}

After washing with PBS, the HPLSCs and frozen sections of sphe- 


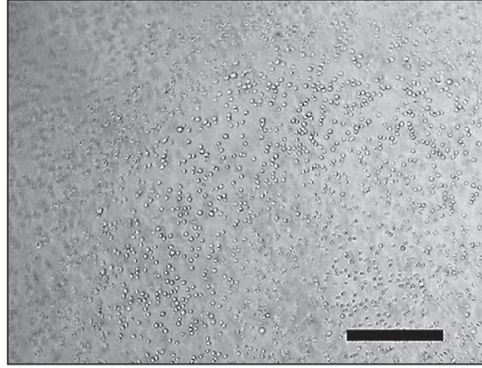

$\mathbf{0 ~ h}$

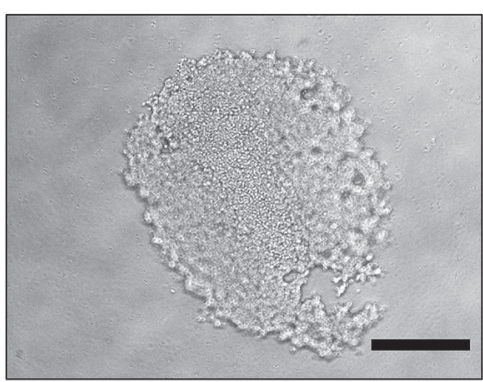

$3 \mathbf{h}$

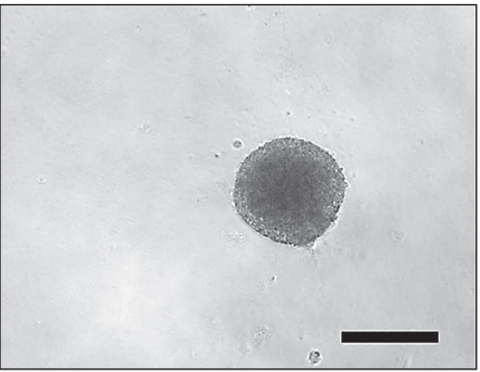

$24 \mathrm{~h}$

Figure 1. Spheroid formation of HPLSCs. Representative phase-contrast images of HPLSC spheroids at 0,3 , and $24 \mathrm{~h}$. The process of spheroid formation is divided into three phases: cell dispersion, cell aggregation, and spheroid compaction. Scale bars $=500 \mu \mathrm{m}$.

A

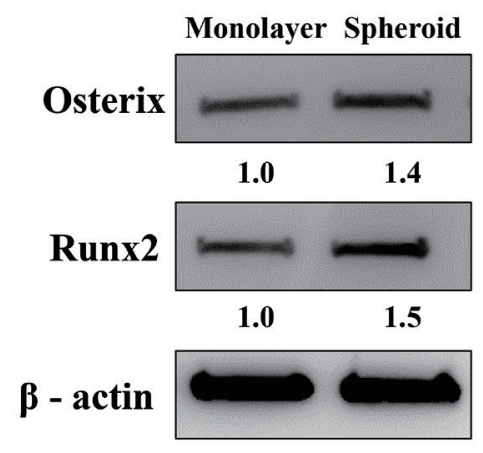

B

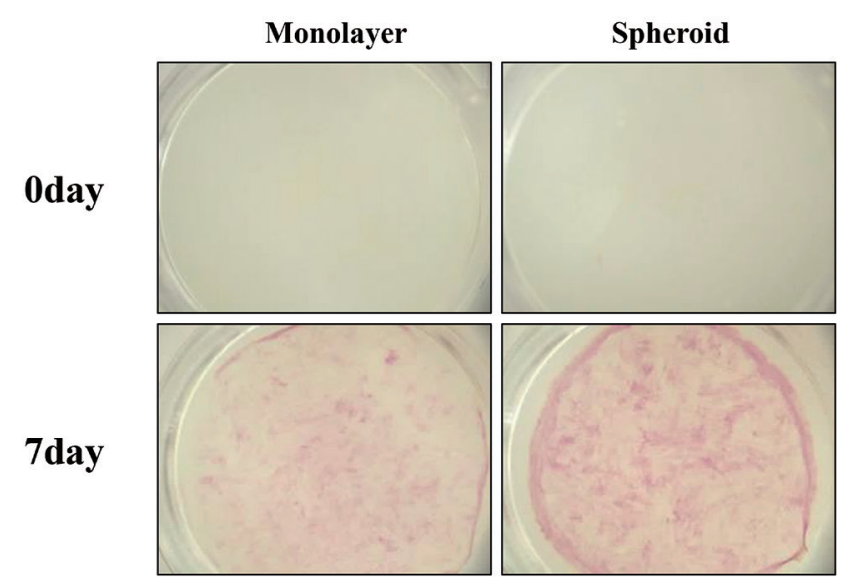

Figure 2. Enhancement of osteogenic differentiation of HPLSC spheroids. A: Western blot analysis of Runx2 and osterix expression of monolayer- and spheroid-derived HPLSCs treated with or without OIM for 7 days. $\beta$-actin was used as a loading control. B: Staining for ALP activity in monolayer- and spheroid-derived HPLSCs cultured with OIM for 0 and 7 days.

roids were fixed with $4 \%$ paraformaldehyde for $10 \mathrm{~min}$ at room temperature (RT), permeabilized with $0.5 \%$ Triton-X in PBS for $10 \mathrm{~min}$, then blocked with $10 \%$ normal goat serum at RT for 10 min and incubated at $4^{\circ} \mathrm{C}$ with primary Abs (dilution, 1:100). After three washes with PBS for 5 min each, the cells were incubated with a combination of secondary Abs with Alexa Flour 488 or 568 (1:200) and Hoechst $33342(5 \mu \mathrm{g} / \mathrm{ml})$, as the nuclear stain (blue), at RT for $45 \mathrm{~min}$. The stained cells were mounted with ProLong Gold Antifade Mountant (Invitrogen Corporation) and viewed under a light microscope (Keyence Corporation of America, Elmwood Park, NJ, USA).

\section{Results \\ Generation of HPLSC spheroids \\ HPLSC spheroids were generated in low-binding plates that can generate a single spheroid per well. The spheroid formation process consists of three phases (Fig. 1). After the HPLSCs were dispersed in the plates at $0 \mathrm{~h}$ (" cell dispersion"), they had spontaneously and loosely aggregated in the medium at approximately $3 \mathrm{~h}$ ("cell aggregation"). Cell aggregates began to generate a compact accumulation. Finally, HPLSCs formed compact spheroids after $24 \mathrm{~h}$ ("spheroid compaction").}

\section{HPLSC spheroid formation enhances osteogenic differentiation}

We assessed the expression levels of osteogenic proteins and ALP staining in monolayer- and spheroid-derived HPLSCs to elucidate the effect of OIM on spheroid culture systems of these cells. Osterix and Runx 2 are known markers of an early phase of osteogenesis. We first examined whether expression levels of both proteins were up-regulated in spheroid-derived HPLSCs cultured with OIM. As demonstrated by western blot analysis, spheroid-derived HPLSCs cultured with OIM showed enhanced expression levels of osterix and Runx 2 on day 7 , as compared with monolayered HPLSCs (Fig. 2A). In accordance with the western blot results, the intensity of ALP staining in spheroid-derived HPLSCs was increased on day 7 , whereas there was no change in the staining intensity of ALP activity in both the monolayer- and spheroid-derived HPLSCs on day 0 (Fig. 2B). These findings indicate that osteogenic differentiation was enhanced in HPLSC spheroids with OIM.

\section{Integrin signaling through FAK is accelerated in HPLSC spheroids}

We next investigated whether the integrin signaling pathway was activated in HPLSC spheroids by immunocytochemical and western blot analysis of FAK. Immunocytochemical detection of integrin $\beta$ and p-FAK was assessed in monolayer- and HPLSC spheroids (Fig. 3A). Integrin $\beta$ expression was observed in the membranes of HPLSC spheroids whereas monolayered cells showed no staining. Remarkable staining of p-FAK was also revealed by HPLSC spheroids as compared with monolayered cells. In accordance with the immunocytochemical results, 
A

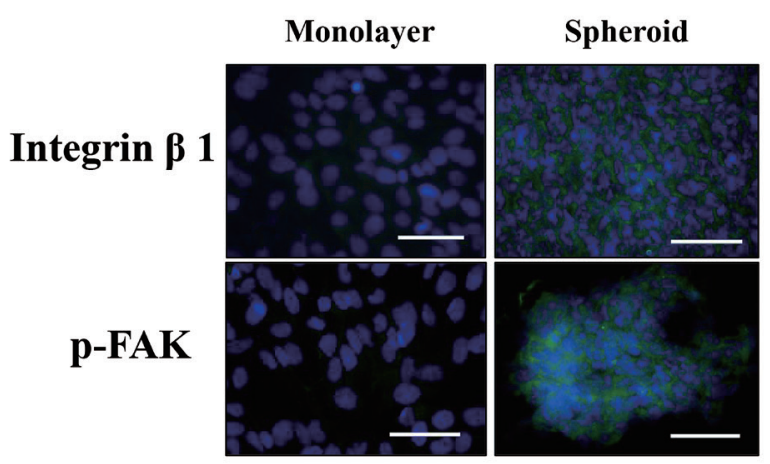

B

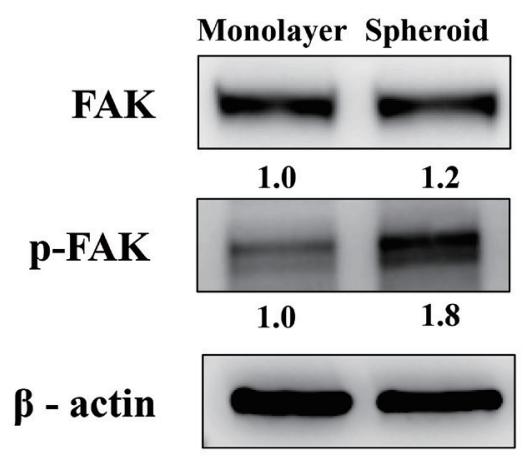

Figure 3. Activation of the integrin signaling pathway in HPLSC spheroids. A: Representative images of immunocytochemical detection of integrin $\beta 1$ and p-FAK in monolayer- and spheroid-cultured HPLSCs cultured with OIM for 1 week.

Scale bar $=50 \mu \mathrm{m}$. B: Western blot analysis of FAK and phosphorylated FAK in monolayer- and spheroid-cultured HPLSCs cultured with OIM for 1 week. $\beta$-actin was used as a loading control. Similar results were obtained in three independent experiments.

A

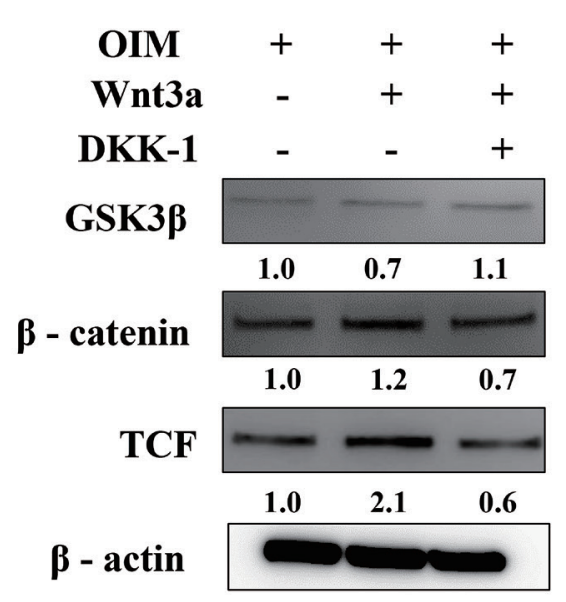

B

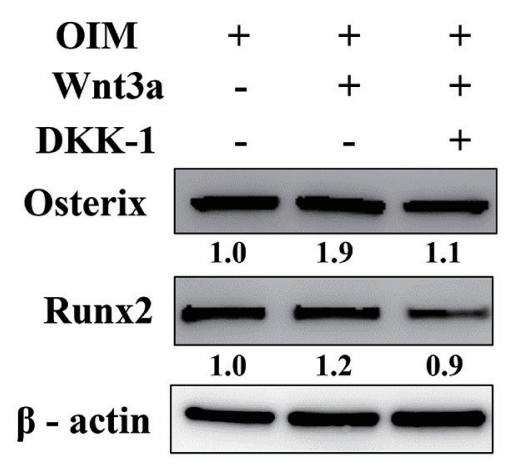

C the expression levels of p-FAK were increased in HPLSC spheroids (Fig. 3B). These findings suggest that during spheroid formation, the integrin signaling pathway may be activated through FAK in HPLSCs composed of spheroids.

Wht 3 a stimulation enhances canonical Wnt signaling-mediated osteogenic differentiation of HPLSC spheroids

The results presented in Fig. 3 prompted us to examine the susceptibility of HPLSC spheroids to Wnt 3a stimulation because enhancement of the integrin/FAK signaling pathway can induce activation of the ca- nonical Wnt/ $\beta$-catenin pathway. Therefore, we first investigated whether Wnt 3 a treatment can accelerate activation of the Wnt/ $\beta$-catenin pathway through GSK-3 $\beta$, $\beta$-catenin, and FAK. As shown in Fig. 4A, Wnt $3 \mathrm{a}$ induced an increase in the expression levels of $\beta$-catenin and TCF, which was effectively decreased by DKK-1, a Wnt antagonist. In contrast, GSK- $3 \beta$ expression was slightly decreased in HPLSC spheroids treated with Wnt 3a (Fig. 4A). These findings suggest that Wnt 3a stimulation may induce the enhancement of canonical Wnt/ $\beta$-catenin pathway through the attenuation of GSK-3 $\beta$ activity. We next examined the effect of Wnt 3a stimulation on enhanced osteogenic differentiation of 
HPLSC spheroids. Increased expression levels of osterix and Runx2 were observed in HPLSC spheroids treated with Wnt 3a, which was effectively decreased by the addition of Dkk-1 (Fig. 4B). As shown in Fig. $4 \mathrm{C}$, Wnt 3a also induced a drastic increase in ALP staining in HPLSC spheroids. Wnt 3a-induced ALP activity was decreased in HPLSC spheroids treated with a combination of Wnt $3 \mathrm{a}$ and Dkk-1 (Fig. 4C). These findings indicate that HPLSC spheroids are susceptible to Wnt 3a stimulation, which results in enhanced osteogenic differentiation of HPLSC spheroids through the activity of the canonical Wnt/ $\beta$-catenin pathway.

\section{Discussion}

A number of three-dimensional cell culture models have been explored, because spheroid cultures particularly allow cells to adapt a native morphology, thereby promoting greater cell-cell contacts and interactions with the $\mathrm{ECM}^{25}$. Our recent studies demonstrated that MSC spheroids had enhanced potential for osteogenic differentiation, as compared with monolayered MSCs ${ }^{26,27)}$. The results of the present study indicate that the formation of HPLSC spheroids can induce susceptibility to osteogenic differentiation through the canonical Wnt/ $\beta$-catenin signaling pathway, which is enhanced by activation of the integrin/FAK pathway.

We demonstrated that OIM induced the enhancement of osteogenic differentiation of spheroid-derived HPLSCs, as compared with monolayer-derived HPLSCs. MSCs cultured with OIM are characterized by an increase in ALP at early stages, followed by matrix deposition, maturation, and mineralization at later stages ${ }^{28,29)}$. In this study, enhanced staining with ALP was observed in spheroid-derived HPLSCs cultured with OIM. Similarly, spheroid-derived HPLSCs cultured with OIM showed increased expression levels of Runx 2 and osterix, which are known markers of the early stage of osteogenesis ${ }^{27,30,31)}$, by western blot analysis. These findings indicate that, as compared with monolayer-derived HPLSCs, HPLSC spheroids are more susceptible to osteogenic differentiation, resulting in accelerated osteogenic differentiation in the early stage.

On the basis of our results concerning immunocytochemical detection of integrin $\beta 1$ and increased expression levels of p-FAK, we propose that activation of the integrin signaling pathway is induced in HPLSCs during the formation of spheroids. An initial step of spheroid formation is involved in cell-cell contact due to cell aggregation that is mediated through integrin-ECM interactions ${ }^{32}$. In this study, immunocytochemical expression of integrin $\beta 1$ was observed in the cell membrane of HPLSC spheroids, indicating that the formation of HPLSC spheroids is mediated through integrins. The cell binding mediated by integrins leads to the recruitment and phosphorylation of FAK and activation of several kinases ${ }^{16,33}$. FAK is a cytoplasmic tyrosine kinase identified as a key mediator of intracellular signaling by integrins in the regulation of different functions in a variety of cells $\mathrm{s}^{34}$. Our western blot analysis showing up-regulation of p-FAK in HPLSC spheroids support this report. The integrin signal pathway is mediated largely through two receptor-associated kinases, integrin-linked kinase and FAK ${ }^{35,36)}$. Therefore, unlike monolayer-derived cells, HPLSC spheroids elicit activation of the integrin signaling pathway through FAK, which is assumed to be required for osteogenic differentiation of these cells.

Western blot analysis showing up- and down-regulation of $\beta$-catenin and GSK-3 $\beta$ expression, respectively, suggests activation of the canonical Wnt/ $\beta$-catenin pathway in the HPLSC spheroids with the activation of the integrin/FAK pathway. This finding supports a recent study that activation of the integrin pathway accelerates the canonical Wnt/ $\beta$-catening signaling pathway ${ }^{16}$. HPLSC spheroids under control of the acti- vated canonical Wnt pathway must be susceptible to Wnt 3a stimulation. Wnt 3a activates the canonical Wnt pathway through the formation of a complex of Wnt, Frizzled, and LRP5 or LRP6 that promotes the phosphorylation of GSK-3 $\beta$, resulting in inhibition of the kinase activity of GSK-3 $\beta$. Inactivation of GSK-3 $\beta$ facilities accumulation of $\beta$-catenin in the target cells, followed by translocation of accumulated $\beta$-catenin into the nucleus ${ }^{37)}$. In this study, Wnt 3a stimulation resulted in the up-regulation of the expression levels of Runx 2 and osterix, and intense staining of ALP in HPLSC spheroids, indicating that HPLSC spheroids retain the activated canonical Wnt pathway. Dkk-1, a natural antagonist of Wnt signaling, binds to LRP-5/LRP-6, which inhibits translocation of $\beta$-catenin into the nucleus and regulation of cell function ${ }^{38)}$. Our results using a combination of Wnt3a and Dkk-1 revealed effective suppression of the expression of Runs 2 and osterix, as well as decreased staining of ALP. These findings suggest that HPLSC spheroids become susceptible to osteogenic differentiation enhanced by activation of the canonical Wnt/ $\beta$-catenin signaling pathway, which is mediated by the integrin pathway through FAK.

There are three possible limitations to this study. First, there is a lack of direct evidence to define the precious mechanisms of the activated canonical Wnt pathway through activation of the integrin pathway. A recent study revealed that the adaptor protein growth factor receptor-bound-2 (Grb2) mediated synergy of Wnt and integrin signaling ${ }^{39)}$. Grb2 coordinates signaling downstream of integrin/FAK and growth factor receptors to activate race and jnk. Grb2 also activates directly disheveled (Dv12) which is present in the downstream of the Wnt/Frizzled/LRP5 and LRP6 complex. Both jnk and Dvl2, once activated by Grb2, induce the stabilization of $\beta$-catenin, leading to its nuclear translocation. Therefore, we speculate that activation of the canonical Wnt/ $\beta$-catenin signaling pathway in HPLSC spheroids may be mediated by Grb2 downstream of the integrin/FAK pathway. Secondly, although the focus of this study was the role of the canonical Wnt signaling pathway, recent studies postulated that the Wnt calcium-dependent pathway, known as the one of two non-canonical Wnt pathways, is involved in osteoblast differentiation and bone formation ${ }^{40,41}$. The Wnt calcium-dependent pathway relies mainly on Wnt5a to stimulate the intracellular release of calcium to activate calmodulin-dependent kinase II, protein kinase $\mathrm{C}$ (PKC), and calcineurin. PKC activity induced by the non-canonical Wnt pathway is responsible for the enhancement of osteogenic differentiation, as confirmed both in vitro and in vivo. Therefore, further studies are warranted to investigate whether the non-canonical Wnt pathway relates to the process of osteogenic differentiation of HPLSC spheroids. Finally, this study may be limited by the lack of direct evidence to investigate whether HPLSC spheroids treated with Wnt3a can accelerate bone formation in vivo. However, we expect that the implantation of Wnt3a-stimulated HPLSC spheroids will promote bone regeneration in a bone defect model based on evidence of increased ALP staining in vitro.

In conclusion, this study revealed that HPLSC spheroids enhance osteogenic differentiation because cells, composed of spheroids, induce the susceptibility of the canonical Wnt pathway, which is mediated by activation of the integrin/FAK pathway during spheroid formation.

\section{Acknowledgments}

We would like to thank Enago (Academic Proofreading Service; www.enago.jp) for the English language review. This work was supported by JSPS KAKENHI (grant number: 18K09567 to JO) and the Private University Research Branding Project. 
J.Hard Tissue Biology Vol. 28(2): 121-128, 2019

\section{Conflict of Interest}

The authors have no competing interest to declare.

\section{References}

1. Chen Y, Shao JZ, Xiang LX, Dong XJ and Zhang GR. Mesenchymal stem cells: a promising candidate in regenerative medicine. Int J Biochem Cell Biol 40: 815-820, 2008

2. Fujii S, Maeda H, Wada N, Tomokiyo A, Saito M and Akamine A. Investigating a clonal human periodontal ligament progenitor/stem cell line in vitro and in vivo. J Cell Physiol 215: 743-749, 2008

3. Wang L, Shen H, Zheng W, Tang L, Yang Z, Gao Y, Yang Q, Wang C, Duan Y and Jin Y. Characterization of stem cells from alveolar periodontal ligament. Tissue Eng Part A 17: 1015-1026, 2011

4. Trubiani O, Di Primio R, Traini T, Pizzicannella J, Scarano A, Piattelli A and Caputi S. Morphological and cytofluorimetric analysis of adult mesenchymal stem cells expanded ex vivo from periodontal ligament. Int J Immunopathol Pharmacol 18: 13-21, 2005

5. Mueller-Klieser W. Three-dimensional cell cultures: from molecular mechanisms to clinical applications. Am J Physiol 273: 1109-1123, 1997

6. Kunz-Schughart LA, Kreutz M and Knuechel R. Multicellular spheroids: a three-dimensional in vitro culture system to study tumour biology. Int J Exp Pathol 79: 1-23, 1998

7. Desoize B. Contribution of three-dimensional culture to cancer research. Crit Rev Oncol Hematol 36: 59-60, 2000

8. Bates RC, Edwards NS and Yates JD. Spheroids and cell survival. Crit Rev Oncol Hematol 36: 61-74, 2000

9. Robinson EE, Zazzali KM, Corbett SA and Foty RA. Alpha5beta1 integrin mediates strong tissue cohesion. J Cell Sci 116: 377-386, 2003

10. Marcellini S, Henriquez JP and Bertin A. Control of osteogenesis by the canonical Wnt and BMP pathways in vivo: cooperation and antagonism between the canonical Wnt and BMP pathways as cells differentiate from osteochondroprogenitors to osteoblasts and osteocytes. Bioessays 34: 953-962, 2012

11. Casey RC, Burleson KM, Skubitz KM, Pambuccian SE, Oegema TR, Jr., Ruff LE and Skubitz AP. Beta 1-integrins regulate the formation and adhesion of ovarian carcinoma multicellular spheroids. Am J Pathol 159: 2071-2080, 2001

12. Damsky CH and Ilic D. Integrin signaling: it's where the action is. Curr Opin Cell Biol 14: 594-602, 2002

13. Giancotti FG and Ruoslahti E. Integrin signaling. Science. 285: 1028-1032, 1999

14. Schneider GB, Zaharias R and Stanford C. Osteoblast integrin adhesion and signaling regulate mineralization. J Dent Res 80: 15401544, 2001

15. Schwartz MA, Schaller MD and Ginsberg MH. Integrins: emerging paradigms of signal transduction. Annu Rev Cell Dev Biol 11: 549599, 1995

16. Du J, Zu Y, Li J, Du S, Xu Y, Zhang L, Jiang L, Wang Z, Chien S and Yang C. Extracellular matrix stiffness dictates Wnt expression through integrin pathway. Sci Rep 6: 20395, 2016

17. Logan CY and Nusse R. The Wnt signaling pathway in development and disease. Annu Rev Cell Dev Biol 20: 781-810, 2004

18. Nishita M, Hashimoto MK, Ogata S, Laurent MN, Ueno N, Shibuya $\mathrm{H}$ and Cho KW. Interaction between Wnt and TGF-beta signalling pathways during formation of Spemann's organizer. Nature 403: 781-785, 2000

19. Labbe E, Letamendia A and Attisano L. Association of Smads with lymphoid enhancer binding factor $1 / \mathrm{T}$ cell-specific factor mediates cooperative signaling by the transforming growth factor-beta and wnt pathways. Proc Natl Acad Sci U S A 97: 8358-8363, 2000

20. Hoppler S and Moon RT. BMP-2/-4 and Wnt-8 cooperatively pattern the Xenopus mesoderm. Mech Dev 71: 119-129, 1998

21. Hartmann C and Tabin CJ. Dual roles of Wnt signaling during chondrogenesis in the chicken limb. Development 127: 3141-3159, 2000

22. Christian JL. BMP, Wnt and Hedgehog signals: how far can they go? Curr Opin Cell Biol 12: 244-249, 2000

23. Capdevila J and Izpisua Belmonte JC. Patterning mechanisms controlling vertebrate limb development. Annu Rev Cell Dev Biol 17: 87-132, 2001

24. Baker JC, Beddington RS and Harland RM. Wnt signaling in Xenopus embryos inhibits bmp4 expression and activates neural development. Genes Dev 13: 3149-3159, 1999

25. Kapur SK, Wang X, Shang H, Yun S, Li X, Feng G, Khurgel M and Katz AJ. Human adipose stem cells maintain proliferative, synthetic and multipotential properties when suspension cultured as self-assembling spheroids. Biofabrication 4: 025004, 2012

26. Miyaguchi N, Kajiya H, Yamaguchi M, Sato A, Yasunaga M, Toshimitu T, Yanagi T, Matsumoto A, Kido H and Ohno J. Bone morphological protein-2 accelerates osteogenic differentiation in spheroid-derived mesenchymal stem cells. J Hard Tissue Biol 27: 343-350, 2018

27. Yamaguchi Y, Ohno J, Sato A, Kido H and Fukushima T. Mesenchymal stem cell spheroids exhibit enhanced in-vitro and in-vivo osteoregenerative potential. BMC Biotechnol 14: 105, 2014

28. Kaartinen MT, El-Maadawy S, Räsänen NH and McKee MD. Tissue transglutaminase and its substrates in bone. J Bone Miner Res 17: 2161-2173, 2002

29. Cheng SL, Yang JW, Rifas L, Zhang SF and Avioli LV. Differentiation of human bone marrow osteogenic stromal cells in vitro: induction of the osteoblast phenotype by dexamethasone. Endocrinology 134: 277-286, 1994

30. Sato A, Kajiya H, Mori N, Sato H, Fukushima T, Kido H and Ohno J. Salmon DNA Accelerates Bone Regeneration by Inducing Osteoblast Migration. PLoS One 12: e0169522, 2017

31. Toda M, Ohno J, Shinozaki Y, Ozaki M and Fukushima T. Osteogenic potential for replacing cells in rat cranial defects implanted with a DNA/protamine complex paste. Bone 67: 237-245, 2014

32. Cui X, Hartanto Y and Zhang H. Advances in multicellular spheroids formation. J R Soc Interface 14: 20160877, 2017

33. Larsen M, Artym VV, Green JA and Yamada KM. The matrix reorganized: extracellular matrix remodeling and integrin signaling. Curr Opin Cell Biol 18: 463-471, 2006

34. Guan JL. Integrin signaling through FAK in the regulation of mammary stem cells and breast cancer. IUBMB Life 62: 268-276, 2010

35. Mitra SK, Hanson DA and Schlaepfer DD. Focal adhesion kinase: in command and control of cell motility. Nat Rev Mol Cell Biol 6: 56-68, 2005

36. Hehlgans S, Haase M and Cordes N. Signalling via integrins: implications for cell survival and anticancer strategies. Biochim Biophys Acta 1775: 163-180, 2007

37. Gordon MD and Nusse R. Wnt signaling: multiple pathways, multiple receptors, and multiple transcription factors. J Biol Chem 281: 22429-22433, 2006

38. Krishnan V, Bryant HU and Macdougald OA. Regulation of bone mass by Wnt signaling. J Clin Invest 116: 1202-1209, 2006

39. Crampton SP, Wu B, Park EJ, Kim JH, Solomon C, Waterman ML 
and Hughes CC. Integration of the beta-catenin-dependent Wnt pathway with integrin signaling through the adaptor molecule Grb2. PLoS One 4: e7841, 2009

40. Olivares-Navarrete R, Hyzy SL, Hutton DL, Dunn GR, Appert C, Boyan BD and Schwartz Z. Role of non-canonical Wnt signaling in osteoblast maturation on microstructured titanium surfaces. Acta Biomaterialia 7: 2740-2750, 2011
41. Kim JH, Liu X, Wang J, Chen X, Zhang H, Kim SH, Cui J, Li R, Zhang W, Kong Y, Zhang J, Shui W, Lamplot J, Rogers MR, Zhao C, Wang N, Rajan P, Tomal J, Statz J, Wu N, Luu HH, Haydon RC and He TC. Wnt signaling in bone formation and its therapeutic potential for bone diseases. Ther Adv Musculoskelet Dis 5: 13-31, 2013 
J.Hard Tissue Biology Vol. 28(2): 121-128, 2019 